\title{
Conductas violentas en primaria Nivel Básico, Medio Superior y Superior
}

\section{Violence Display in Elementary, High School and University Education}

\author{
GOMEZ FIGUEROA, J. A. ${ }^{1}$ \\ RODRÍGUEZ CERVANTES, V. O. ${ }^{2}$ \\ RIVERA GIRÓN, A. de J. ${ }^{3}$ \\ LOZANO FLORES, J. C. ${ }^{4}$ \\ MOLINA ARRIOLA, J. E. ${ }^{5}$ \\ QUINTANA RIVERA, L. ${ }^{6}$ \\ MONCADA JIMÉNEZ, J. ${ }^{7}$
}

\begin{abstract}
Resumen
El propósito del estudio fue determinar conductas violentas en estudiantes de educación primaria, secundaria y superior. Participaron 1726 estudiantes de instituciones educativas públicas de primaria, secundaria y universidad, quienes completaron el Cuestionario de Agresión. Se encontró que quienes reciben clases de Educación Física muestran menor agresión física ( $p \leq 0.001$ ) y estado de hostilidad ( $p$ $\leq 0.001$ ) en comparación a quienes no recibían clases de Educación Física. Se concluye que quienes se mantienen físicamente activos presentan menos conductas violentas.

Palabras clave: violencia escolar, educación física, agresión.
\end{abstract}

\begin{abstract}
The purpose of the study was to determine violent behavior in students of primary, secondary and higher education. Participants were 1726 students from public primary, secondary and university institutions who completed the Agresion Questionnaire. Those engaged in Physical Education classes showed less physical aggression ( $p \leq 0.001)$ and a state of hostility $(p \leq 0.001)$ compared to those in the Physical Education classes. It is concluded that those who remain physically active have less violent behaviors.
\end{abstract}

Key words: school violence, physical education, agresión.

\section{Introducción}

La violencia es un fenómeno mundial con consecuencias importantes en el ámbito educativo. Se estima que mil millones de niños con edades entre 2 y 17 años han sufrido algún tipo de violencia (Ferrara et al., - (2019). Las conductas agresivas en el ámbito escolar son situaciones conocidas por la mayoría de los estudiantes (Albaladejo-

\footnotetext{
${ }^{1}$ Docente. Facultad de Educación Física, Deporte y Recreación Deportes. Universidad Veracruzana. julgomez@uv.mx

${ }^{2}$ Docente. Facultad de Educación Física, Deporte y Recreación Deportes. Universidad Veracruzana. virodriguez@uv.mx

3 Docente. Facultad de Educación Física, Deporte y Recreación Deportes. Universidad Veracruzana. angelrivera02@uv.mx

${ }^{4}$ Docente. Facultad de Educación Física, Deporte y Recreación Deportes. Universidad Veracruzana. jlozano@uv.mx

${ }^{5}$ Docente. Facultad de Educación Física, Deporte y Recreación Deportes. Universidad Veracruzana. josmolina@uv.mx

${ }^{6}$ Docente. Facultad de Educación Física, Deporte y Recreación Deportes. Universidad Veracruzana. Lquintana@uv.mx

${ }^{7}$ Docente. Escuela de Educación Física y Deportes. Universidad de Costa Rica. jose.moncada@ucr.ac.cr
} 
Blázquez, 2011) y forman parte de la vida diaria en los centros educativos, viéndose todos los escolares implícitos en ellas como agresores, víctimas o espectadores. Se ha observado que un número elevado de estudiantes llega a participar con papeles diferentes en la dinámica y trayectoria de la violencia que se presenta en instituciones educativas, concretamente en la etapa final de educación infantil y el primer ciclo de educación primaria. Sin embargo, recientemente se han descrito conductas violentas en la población de estudiantes universitarios, con manifestaciones principalmente de tipo sexual (Esquivel-Santoveña et al., _2017; Kelmendi \& Baumgartner, (2017).

La violencia física incluye el uso de la fuerza para dañar al otro con acciones como empujones, jalones, pellizcos, rasguños, golpes, bofetadas, patadas y aislamiento. Por su parte, la violencia emocional incluye la manifestación de gritos, insultos, amenazas, prohibiciones, indiferencia, ignorancia, abandono afectivo, celos patológicos, humillaciones, descalificaciones, chantajes, manipulación y coacción (Gobierno de los Estados Unidos Mexicanos, 2012). Los hallazgos de un reciente meta análisis de 114 publicaciones determinaron que cualquier manifestación de violencia escolar afecta negativamente a los estudiantes en su salud mental, rendimiento académico y posible involucramiento en actividades delictivas futuras (Polanin et al., 2020).

En países como México, se sabe que la violencia escolar es un fenómeno frecuente en individuos provenientes de hogares disfuncionales que tienden a tener conductas agresivas que reflejan dentro del ámbito escolar. Rodríguez Abrahantes et al., (2015), reportaron que los factores psicosociales de riesgo asociados a las manifestaciones de conductas violentas se deben en un $28.3 \%$ a quienes tienen padres alcohólicos y en un $24.5 \%$ a quienes viven en situaciones familiares inadecuadas y con violencia intrafamiliar, siendo la violencia verbal la más utilizada con acciones como poner motes molestos, amenazar a otros mediante palabras para causar miedo y obligarlos a realizar cosas, insultarse entre ellos y hablar mal unos de otros. Sin embargo, también se sabe que un ambiente escolar positivo puede reducir las conductas violentas de estudiantes de hogares disfuncionales (Moore et al ( 2020).

Existe evidencia que indica que en el nivel de educación secundaria existen manifestaciones de violencia (Prieto García, 2005). Esta información se recolecta principalmente por medio de cuestionarios, por medio de los cuales se logra analizar la frecuencia con la que los estudiantes consideran que aparecen diferentes tipos de violencia escolar, constituyendo así una herramienta de utilidad a disposición de investigadores y orientadores psicoeducativos para el análisis de la violencia en centros escolares (Álvarez-García et al., (2013). Por ejemplo, en un estudio realizado por Gómez Figueroa y Salazar (2015), se confirmó que existen diferencias significativas en el comportamiento de la agresión verbal, física, estado de ira y hostilidad en la población en nivel de secundaria de Veracruz, México. También se encontraron diferencias significativas en los grupos de edad de 11 a 18 años en las cuatro dimensiones estudiadas y no observaron diferencias por sexo.

Estudios similares y de tipo transcultural se habían descrito anteriormente. Por ejemplo, se compararon 1075 estudiantes de educación secundaria de Valparaíso, Chile, con 637 estudiantes españoles de Asturias y León (Guerra Vio et al., 2011). Se encontró que la muestra de estudiantes chilenos percibió una mayor frecuencia de violencia física que los estudiantes españoles. En otro estudio del mismo nivel educativo se encuestó a 2597 estudiantes Asturianos, entre los cuales identificaron un alto nivel de violencia verbal (Alvarez García et al., (2014).

El reporte de un estudio realizado en una institución de educación secundaria de la Universidad de Guadalajara, México, en la que se evaluaron a 603 alumnos de los diferentes turnos escolares, se encontró que el 71.8\% evidenció violencia, dentro de la cual destacan los insultos (22.6\%) y los golpes (21.1\%), no habiendo diferencias significativas en la percepción de la violencia entre hombres y mujeres; es decir, las percepciones fueron similares en relación a la violencia escolar y los estudiantes coinciden en que la escuela es un espacio que les permitiría obtener aprendizajes significativos desde el currículo sobre violencia (Vázquez Valls et al., - (2005). Otro estudio 
realizado en México con 12937 estudiantes de secundaria reportaron manifestaciones y factores asociados al acoso escolar (i.e., agresión física y verbal), en el que 3.1\% presenta algún tipo de agresión, con mayor frecuencia entre los hombres, entre quienes la manifestación más utilizada es la de tipo verbal (7.2\%) (Martínez Vilchis et al., 2015).

La violencia y el acoso escolar son dos graves problemas que enfrentan millones de estudiantes en el mundo. Ramos-Rodríguez y Aranda-Beltrán (2020) realizaron un estudio descriptivo, transversal y analítico en estudiantes universitarios de primer semestre de un Centro Universitario de Ciencias de la Salud de México, con el objetivo de analizar la relación entre violencia y acoso escolar con el sexo y edad de los estudiantes, obteniendo como resultado que el género masculino y de más de 20 años de edad padecen en mayor medida de violencia escolar, los principales tipos de violencia y acoso fueron las agresiones verbales y la exclusión social.

A pesar de que existen evidencias previas de estudios acerca de la violencia en instituciones educativas en México, no se han abordado comparaciones entre los diversos niveles de escolaridad. En este contexto, el propósito del presente estudio fue comparar cuatro dimensiones de violencia en estudiantes de primaria, secundaria y universidad mexicanas. La hipótesis que guía el estudio es que la frecuencia de las dimensiones de violencia era similar entre los diferentes niveles educativos.

\section{Metodología}

\subsection{Diseño y participantes}

El diseño del estudio fue de tipo descriptivo transversal (Hernández et al., (2010) y buscó comparar cuatro dimensiones de la violencia en estudiantes de tres niveles educativos en México. Los voluntarios fueron estudiantes del Estado de Veracruz, México. El muestreo fue por conveniencia y estuvo conformado por 1726 estudiantes inscritos a planteles educativos del sector público. Los niveles participantes fueron primaria $(\mathrm{n}=$ 814 ), secundaria ( $n=137$ secundaria y $n=506$ bachillerato) y universidad $(n=269)$.

\subsection{Instrumentos}

Se diseñó una hoja de recolección de datos que contenía información demográfica (e.g., edad, sexo del participante, cantidad de hermanos), así como de posibles variables asociadas a las dimensiones de violencia (e.g., cantidad de tiempo viendo televisión, cantidad de tiempo jugando videojuegos, tiempo solitario) y relacionadas con la Educación Física y los deportes (e.g., reciben clases de Educación Física, se han lesionado en clases de Educación Física, práctica de deportes).

Se utilizó el instrumento "Agression Questionnaire" (Buss \& Perry, 1992), en su versión española reducida (Morales-Vives et al., 2005) para medir el constructo de violencia. La elección del instrumento estuvo considerada por la validación y recurrencia en los estudios de este tipo, además de sus índices psicométricos. Se sabe que el cuestionario ha sufrido modificaciones a lo largo de varias décadas; sin embargo, en la actualidad es uno de los formatos con mayor aplicabilidad en los jóvenes y adolescentes (Gómez Figueroa \& Salazar, 2015). EI instrumento está conformado por 29 ítems en escala Likert agrupados en cuatro dimensiones: a) agresión verbal, b) agresión física, c) ira, y d) hostilidad. Cada dimensión se cataloga en tres niveles: a) bajo, b) medio, y c) alto. Los participantes completaron un formulario de consentimiento informado escrito de los padres de familia o tutor y director(a) del centro educativo. La recolección de datos se efectuó en el horario de la clase de Educación Física para los niveles de primaria y secundaria, para el bachillerato y la universidad en horario entre clase, en un lugar limpio, iluminado y ventilado. Las dudas que surgieron fueron aclaradas por el investigador. 


\subsection{Análisis estadístico}

Los análisis se realizaron con el IBM SPSS Statistics, versión 22.0 (Armonk, NY, USA). Los valores se resumen como la media y la desviación estándar $(\mathrm{M} \pm \mathrm{DE}$ ) para las variables continuas, así como frecuencias y porcentajes para las variables categóricas. Se calcularon pruebas t-student para grupos independientes para comparar los promedios de las variables agresión física, agresión verbal, estado de ira y estado de hostilidad. Se utilizaron pruebas de ANOVA de una vía para comparar los promedios de las variables entre variables con más de dos categorías. Se utilizó la prueba de post-hoc de Tukey cuando la prueba ómnibus de ANOVA fue significativa. Finalmente, se calcularon pruebas de correlación de Pearson entre las variables continuas del estudio. La significancia estadística se estableció en $\mathrm{p} \leq 0.05$.

\section{Resultados}

Se recolectaron datos de 1726 estudiantes de primaria, secundaria (secundaria y bachillerato) y universidad. En la tabla 1 se presentan las estadísticas descriptivas de los tres niveles de las cuatro dimensiones de violencia escolar por nivel educativo.

Tabla 1

Estadística descriptiva de las dimensiones de violencia por nivel educativo ( $n=1726)$. Los valores representan las frecuencias (f) y su respectiva proporción (\%).

\begin{tabular}{|c|c|c|c|c|c|c|c|c|c|c|}
\hline \multirow[b]{2}{*}{ Dimensión y Nivel } & \multicolumn{2}{|c|}{ Primaria } & \multicolumn{2}{|c|}{ Secundaria } & \multicolumn{2}{|c|}{ Bachillerato } & \multicolumn{2}{|c|}{ Universidad } & \multicolumn{2}{|c|}{ Total } \\
\hline & $f$ & $\%$ & $f$ & $\%$ & $f$ & $\%$ & $f$ & $\%$ & $f$ & $\%$ \\
\hline \multicolumn{11}{|l|}{ Agresión Física } \\
\hline Bajo & 108 & 6.5 & 17 & 1.0 & 57 & 3.4 & 29 & 1.7 & 211 & 12.7 \\
\hline Medio & 404 & 24.2 & 68 & 4.1 & 272 & 16.3 & 168 & 10.1 & 912 & 54.7 \\
\hline Alto & 302 & 18.1 & 52 & 3.1 & 117 & 7.0 & 72 & 4.3 & 543 & 32.6 \\
\hline \multicolumn{11}{|l|}{ Agresión Verbal } \\
\hline Bajo & 480 & 28.8 & 56 & 3.4 & 233 & 14.0 & 146 & 8.8 & 915 & 54.9 \\
\hline Medio & 312 & 18.7 & 78 & 4.7 & 209 & 12.5 & 121 & 7.3 & 720 & 43.2 \\
\hline Alto & 23 & 1.4 & 3 & 0.2 & 4 & 0.2 & 2 & 0.1 & 32 & 1.9 \\
\hline \multicolumn{11}{|l|}{ Estado de Ira } \\
\hline Bajo & 95 & 5.7 & 8 & 0.5 & 36 & 2.2 & 27 & 1.6 & 166 & 10.0 \\
\hline Medio & 514 & 30.8 & 93 & 5.6 & 316 & 19.0 & 198 & 11.9 & 1121 & 67.3 \\
\hline Alto & 206 & 12.4 & 36 & 2.2 & 94 & 5.6 & 44 & 2.6 & 380 & 22.8 \\
\hline \multicolumn{11}{|l|}{ Estado de Hostilidad } \\
\hline Bajo & 227 & 13.6 & 33 & 2.0 & 163 & 9.8 & 129 & 7.7 & 552 & 33.1 \\
\hline Medio & 428 & 25.7 & 90 & 5.4 & 251 & 15.1 & 135 & 8.1 & 904 & 54.2 \\
\hline Alto & 160 & 9.6 & 14 & 0.8 & 32 & 1.9 & 5 & 0.3 & 211 & 12.7 \\
\hline
\end{tabular}

La frecuencia de niveles de agresión física reportados por los estudiantes de todos los niveles educativos fueron predominantemente moderados. La frecuencia de niveles de agresión física para toda la muestra también fue moderada. La frecuencia de niveles de agresión verbal de los estudiantes de nivel primaria y universitarios fueron bajos, y en los niveles de secundaria y bachillerato fueron moderados. La frecuencia de niveles de agresión física para toda la muestra fue baja. La frecuencia de niveles de ira de los estudiantes de primaria, secundaria, bachillerato y universidad fueron moderados. La frecuencia de los niveles de ira para toda la muestra fue moderada. Finalmente, la frecuencia de los niveles de hostilidad de los estudiantes de primaria, secundaria, bachillerato y universidad fueron moderados. La frecuencia de los niveles totales de hostilidad para toda la muestra fue moderada. 
Se encontró que quienes reciben clases de Educación Física mostraron menores puntajes de agresión física ( $p \leq$ 0.001 ) y estado de hostilidad ( $\leq \leq 0.001$ ) en comparación a quienes no recibían clases de Educación Física (Figura 1A). No se encontraron diferencias estadísticamente significativas para las dimensiones de agresión verbal $(p=$ 0.530 ) y estado de ira $(p=0.719)$. Se encontró que quienes desean recibir clases de Educación Física mostraron mayores puntajes de agresión física $(p \leq 0.001)$ en comparación a quienes no desean recibir clases de Educación Física (Figura 1B). No se encontraron diferencias estadísticamente significativas para las dimensiones de agresión verbal $(p=0.165)$, estado de ira $(p=0.075)$ y estado de hostilidad $(p=0.083)$. Se encontró que quienes se han lesionado en las clases de Educación Física poseen puntajes menores en las dimensiones de agresión verbal $(p=$ $0.004)$, estado de ira $(p=0.011)$ y estado de hostilidad $(p \leq 0.001)$ que quienes no se han lesionado en las clases de Educación Física (Figura 1C). No se encontró una diferencia estadísticamente significativa en la dimensión de agresión física $(p>0.05)$.

Figura 1

1A) Diferencias en las dimensiones de violencia entre quienes reciben y quienes no reciben clases de Educación Física. 1B) Diferencias en las dimensiones de violencia entre quienes desean recibir y quienes no desean recibir clases de Educación

Física. 1C) Diferencias en las dimensiones de violencia entre quienes se han lesionado y quienes no se han lesionado en las clases de Educación Física. Los valores son $\mathrm{M} \pm \mathrm{DE}$. ${ }^{*} \mathrm{p} \leq 0.001$ entre Si y No en cada variable.

$\mathbf{A}$

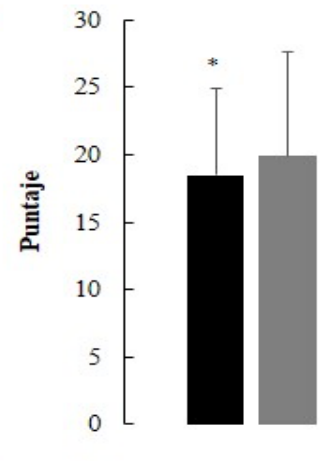

B

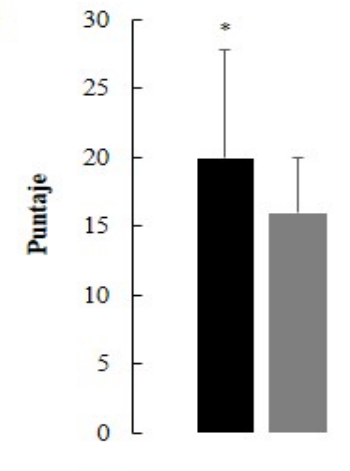

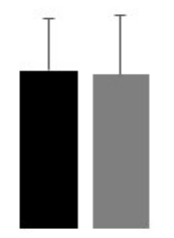

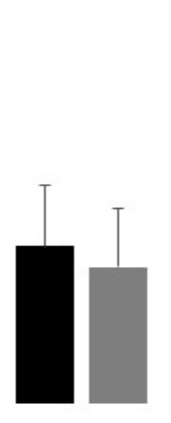

$\mathbf{C}$

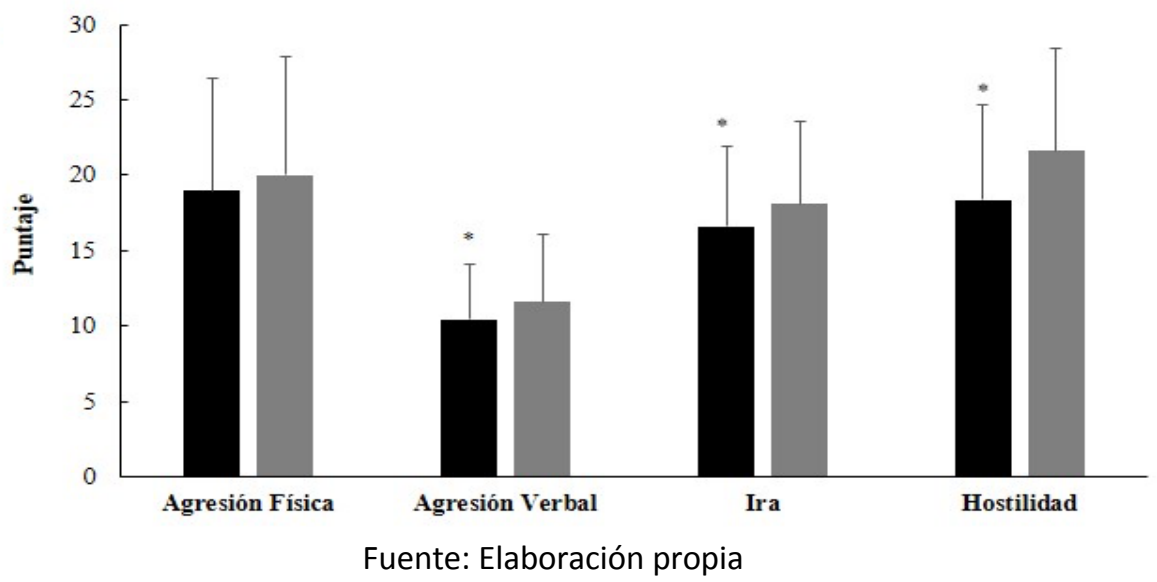

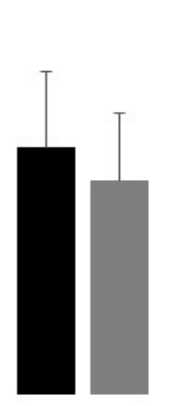
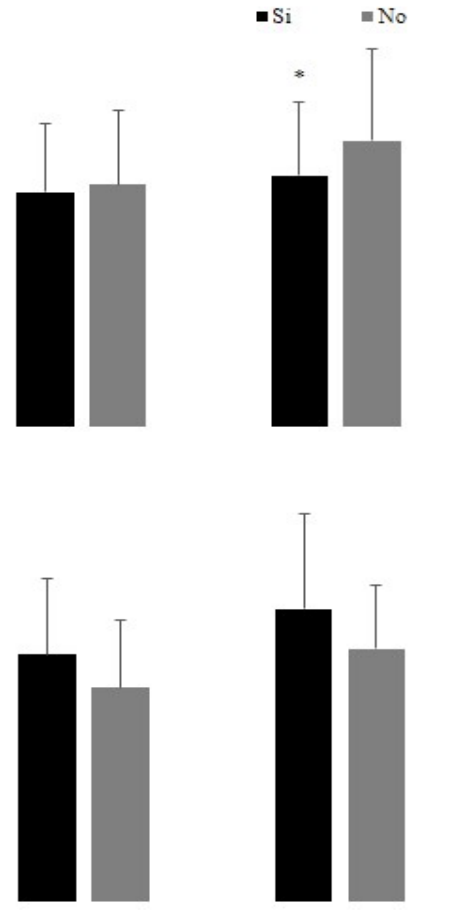
Se encontró que quienes regularmente realizan deporte poseen puntajes menores en la dimensión de agresión física que quienes no realizan actividades deportivas ( $p \leq 0.001$; Figura $2 A$ ). Sin embargo, no se encuentran esas diferencias en las dimensiones de agresión verbal $(p=0.547)$, estado de ira $(p=0.584)$ y estado de hostilidad ( $p$ $=0.354)$. Se encontró que quienes juegan videojuegos muestran puntajes mayores en las dimensiones de agresión física ( $p \leq 0.001)$, agresión verbal $(p \leq 0.001)$ y estado de hostilidad $(p \leq 0.001)$ que quienes no juegan videojuegos (Figura 2B). No se encontró una diferencia estadísticamente significativa en la dimensión de estado de ira $(p>0.05)$.

Figura 2

2A) Puntajes en las variables entre quienes regularmente realizan deporte y quienes no lo hacen. 2B) Diferencias en las dimensiones de violencia entre quienes juegan y quienes no juegan videojuegos. Los valores son $M \pm D E .{ }^{*} p \leq 0.001$ entre Si y No en cada variable

$\mathbf{A}$

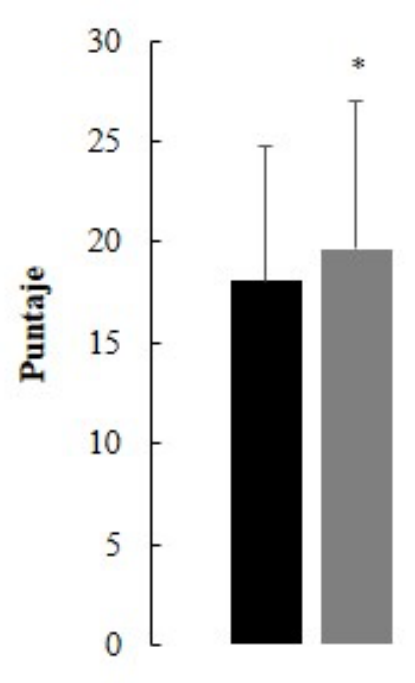

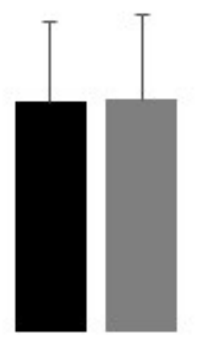

B

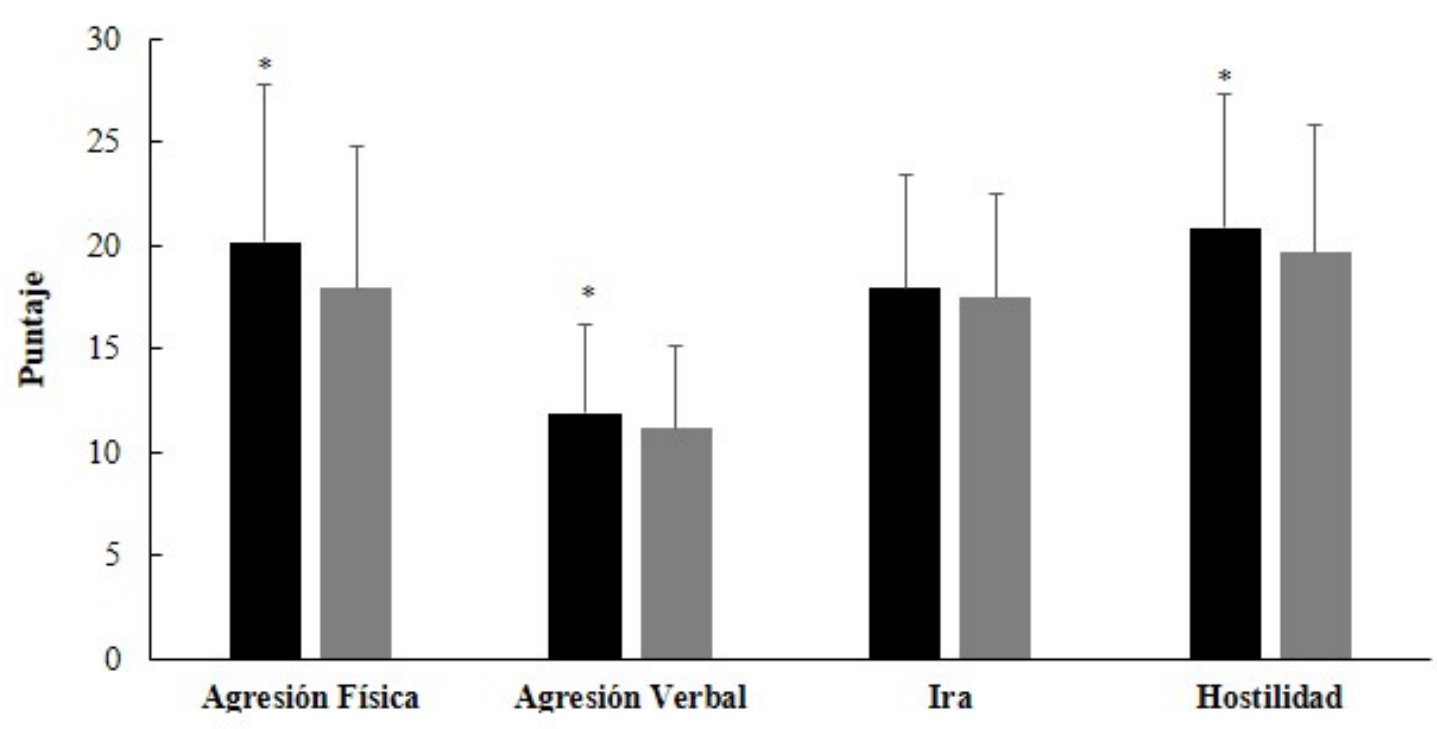

- $\mathrm{Si} \quad \mathrm{No}$
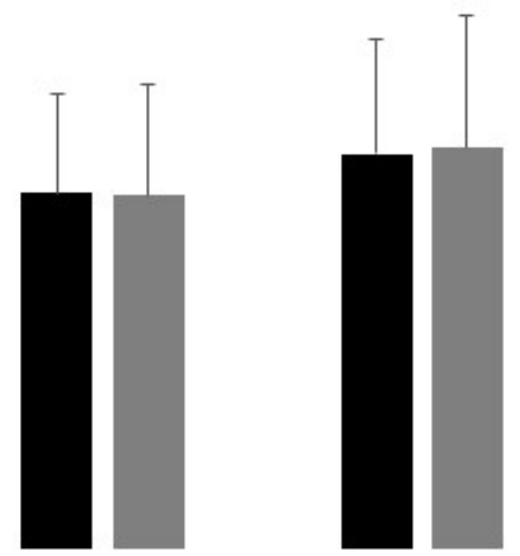

Fuente: Elaboración propia

Se encontraron diferencias significativas en los estados de soledad en las dimensiones de agresión física ( $p \leq$ $0.001)$, agresión verbal ( $p \leq 0.001)$, estado de ira $(p \leq 0.001)$ y estado de hostilidad $(p \leq 0.001)$. El análisis posthoc reveló que para cada variable se observan mayores puntajes a medida que la persona pasa más tiempo sin compañía (Figura 3A). Se encontraron diferencias significativas en la cantidad de tiempo dedicado a jugar videojuegos en las dimensiones de agresión física $(p \leq 0.001)$, agresión verbal $(p \leq 0.001)$, estado de ira $(p=0.001)$ 
y estado de hostilidad ( $\mathrm{p} \leq 0.001$ ). El análisis post-hoc reveló que para cada variable se observan mayores puntajes a medida que la persona pasa más tiempo jugando videojuegos (Figura 3B).

Figura 3

3A) Puntajes en las variables en función del grado de soledad del participante. 3B) Puntajes en las variables en función de la cantidad de días en que los participantes jugaban videojuegos. Los valores son $M \pm D E$. Las letras diferentes en cada variable representan diferencias estadísticamente significativas en el post-hoc a $p \leq 0.05$.

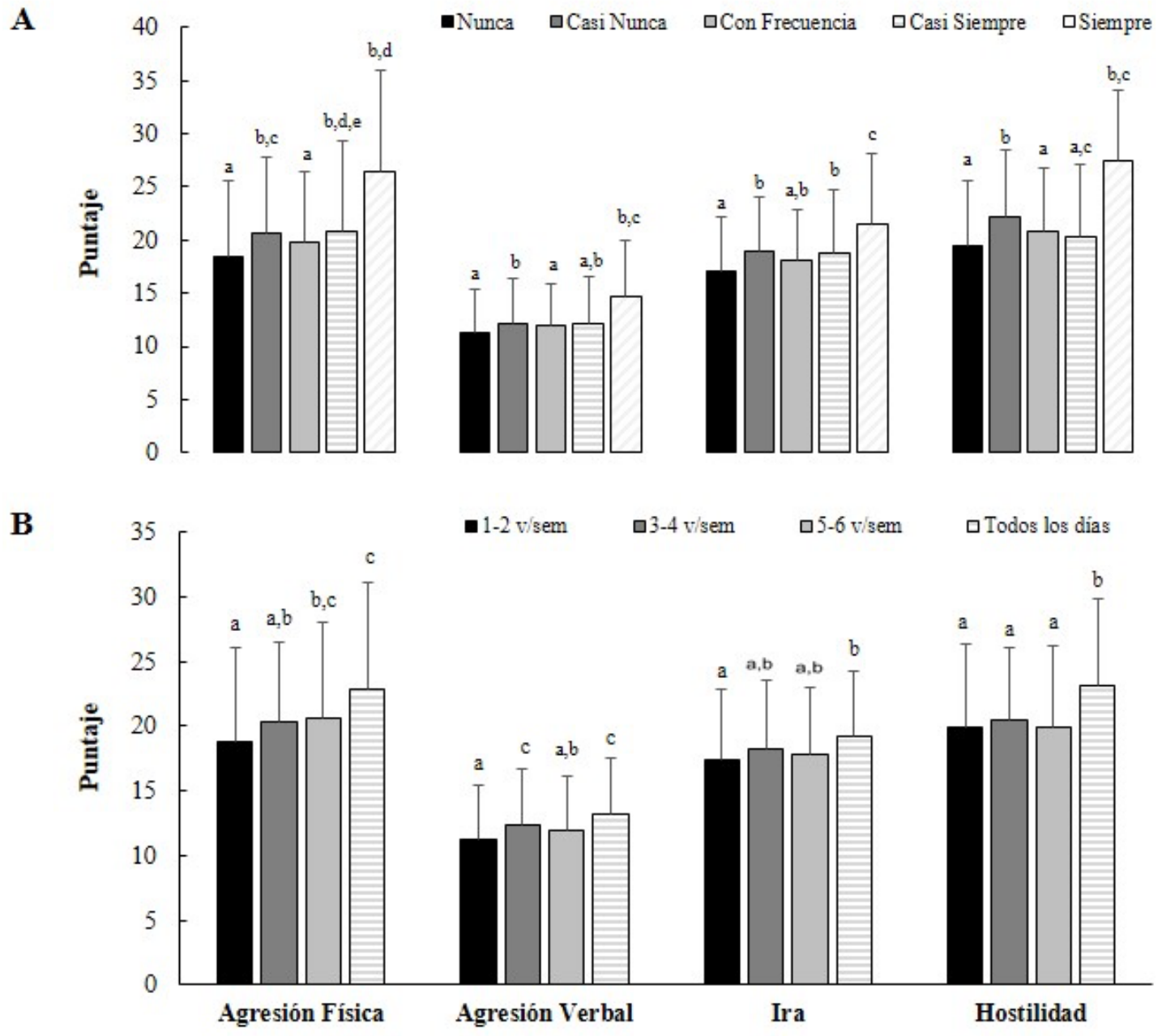

Fuente: Elaboración propia

Se encontró que a mayor edad de los estudiantes se reducían significativamente los valores de los puntajes en las dimensiones de agresión física ( $r=-0.10, p \leq 0.001)$, estado de ira $(r=-.07, p \leq 0.010)$, y estado de hostilidad $(r=-0.22, p \leq 0.001)$. También se encontró que a mayor edad de los participantes, menor era la cantidad de horas de televisión diarias $(r=-0.55, p \leq 0.001)$. La cantidad de horas diarias reportadas en ver televisión se asoció con mayores puntajes de agresión física $(r=0.08, p \leq 0.001)$, agresión verbal $(r=0.06, p \leq 0.050)$, estado de ira $(r=$ $0.07, p \leq 0.010)$ y estado de hostilidad $(r=0.17, p \leq 0.001)$. Finalmente, se encontró que a mayor cantidad de hermanos también aumentaban los puntajes de las dimensiones de agresión física $(r=0.07, p \leq 0.010)$ y estado de hostilidad $(r=0.07, p \leq 0.010)$. 


\section{Conclusiones}

El propósito del presente estudio fue comparar cuatro dimensiones de violencia en estudiantes mexicanos de primaria, secundaria y universidad. Se sabe que la violencia escolar puede generar trastorno de apego reactivo, inactividad física moderada, sobrepeso u obesidad, diabetes, hábitos de fumar, consumo excesivo de alcohol, mala salud autoevaluada, cáncer, enfermedades cardíacas y respiratorias (Ferrara et al., 2019). En el presente estudio se confirma la hipótesis inicial, ya que se obtuvieron niveles moderados de agresión física, estado de ira y estado de hostilidad, así como niveles bajos de agresión física para toda la muestra. La dimensión de la agresión verbal en nivel primaria se ha comportado con el paso del tiempo de manera homogénea en los estudiantes mexicanos, ya que Gómez Figueroa \& Salazar (2015) reportaron un 69.3\% de niveles bajos de agresión verbal, mientras que en el presente estudio se reportan un $54.9 \%$.

En otros países, la agresión verbal es altamente frecuente. Por ejemplo, en un estudio comparativo en el que participaron 2582 estudiantes de China, Hong Kong y Taiwán (J. K. Chen \& Chen, 2020), se encontró que la violencia verbal es más frecuente que otras formas de agresión, especialmente entre los hombres. Sin embargo, no se encontraron diferencias entre los distintos niveles educativos, algo similar a lo encontrado en el presente estudio.

Los bajos niveles de la dimensión de agresión física reportados previamente (Gómez Figueroa \& Salazar, 2015) fueron del $30.7 \%$, lo cual contrasta con lo encontrado en el presente estudio (12.7\%). Es decir, en una muestra de participantes similares, los niveles de agresión física se redujeron en aproximadamente 41\%; sin embargo, no se pueden explicar las posibles causas de esta disminución. Se podría especular que en cinco años se han desarrollado mayores estrategias globales para fomentar la conciencia social acerca de la importancia de reducir la violencia en el ámbito escolar (Cornell, 2020; Q. Chen et al.,_2020; Taylor, 2020). Por su parte, la dimensión del estado de ira muestra una diferencia significativa entre los estudiantes de nivel básico y los de nivel superior. Este hallazgo es similar al reportado anteriormente (Gómez Figueroa \& Salazar, 2015).

Los universitarios analizados muestran una mayor prevalencia de niveles medios en su estado de ira, siendo esta la mayor dimensión de violencia escolar que se observa en este nivel educativo, mientras que la agresión verbal es baja. Esto contrasta con los resultados obtenidos por Ramos-Rodríguez y Aranda-Beltrán (2020), quienes argumentan que los principales tipos de violencia son agresiones verbales y la exclusión social. Se coincide con Gázquez Linares et al., (2008), quienes analizaron que la percepción de universitarios sobre el origen de la violencia escolar es un factor de la escasa educación en el respeto a los demás. Lo encontrado en el presente estudio en el nivel universitario, evidencia que la agresión física se ubica en niveles medios, la agresión verbal en niveles bajos, los estados de ira en niveles medios, al igual que los de hostilidad. En otro estudio se evaluó a 377 universitarios de la universidad de Huelva, España, mostrando una mayor prevalencia en agresión verbal entre los alumnos, aunque también se señala el uso de las redes sociales para insultar y hablar mal del profesorado (Conde Vélez \& Delgado-García, 2020).

En el presente estudio se encontraron variables que posiblemente moderan las dimensiones de la violencia en el ámbito escolar. Por ejemplo, los estudiantes que reciben clases de Educación Física reportaron puntajes menores de agresión física y estado de hostilidad comparados con quienes no recibían clases. La actividad y especialmente el deporte han demostrado cambios positivos (i.e., reducción) en la agresión verbal, física e ira en adolescentes entre 14 y 16 años (Trajković, et al., (2020); Trajković et al (2020b). Este hallazgo es relevante porque en el presente estudio quienes indicaron que deseaban recibir clases de Educación Física también mostraron mayores puntajes de agresión física, por lo que implementar son solamente clases de Educación Física sino también entrenamiento en deportes crónicamente pueden ayudar a reducir diferentes manifestaciones de violencia escolar (Park et (2017; Shachar et al., +2016). 
Es posible que las clases de Educación Física permitan a los estudiantes ser más resilientes (San Román-Mata et al., (2020) o que la práctica de deportes les permita poseer una mayor fortaleza mental (Stamatis et al., 2020), ya que en el presente estudio se encontró que incluso aquellos que se habían lesionado durante las clases de Educación Física presentaron puntajes menores en las dimensiones de agresión verbal, estado de ira y estado de hostilidad que quienes no se habían lesionado durante las clases. Por lo tanto, es posible que quienes realizan actividad física estructurada puedan administrar mejor sus emociones y que este beneficio se mantenga a largo plazo (i.e., efecto crónico).

En este estudio se encontró que el uso de videojuegos y la cantidad de tiempo utilizándolos se asociaba con mayor comportamiento violento (i.e., agresión física, agresión verbal, estado de hostilidad). Se han reportado investigaciones que apoyan la noción de que los videojuegos promueven el comportamiento agresivo y violento (Madrigal-Pana et al., (2018); sin embargo, no hay conclusiones definitivas al respecto. Al menos con la información recopilada en el presente estudio, se observa una clara diferencia en el comportamiento violento entre quienes utilizan videojuegos y quienes no lo hacen (Figura 2B).

Otro elemento que puede explicar el comportamiento violento es la soledad. En el presente estudio se encontró que quienes pasan más tiempo en soledad también muestran mayores puntajes de agresión física, agresión verbal, estado de ira y estado de hostilidad. El tema de la soledad y la asociación con la violencia es complejo y particular para cada sociedad. En México, se ha pensado en comprender el fenómeno integrando las áreas psicológicas, cognitivas, neuroendocrinas e inmunológicas, así como el funcionamiento social, de salud física y genética (Jenkin et al.s, (2020). Por lo tanto, para efectos del presente estudio, lo que se encuentra es una mayor frecuencia de comportamiento violento cuando la persona permanece más tiempo en soledad, lo cual podría ser atenuado a través de la incorporación de los estudiantes en las clases curriculares y extracurriculares de deportes y actividad física.

En el presente estudio se encontraron algunas correlaciones relevantes. Por ejemplo, a mayor edad, hubo un menor comportamiento violento. Es posible que las experiencias vividas por los estudiantes les permitan manejar mejor sus emociones, lo cual se podría traducir en un menor comportamiento violento debido a las posibles consecuencias legales o morales. También se encontró que a mayor cantidad de hermanos, hubo una mayor cantidad de comportamiento violento, lo cual se puede explicar parcialmente por factores asociados a situaciones violentas que ocurre como parte de la dinámica familiar (Ingram et al., f 2020; Rodríguez Abrahantes et al., 2015).

En conclusión, como se percibe en los resultados y discusión de la presente investigación que aborda la problemática de la violencia escolar desde el nivel de primaria hasta el universitario, el comportamiento de las dimensiones que conforman a la violencia escolar se pueden abordar desde un enfoque social. Sin embargo, el diseño de estrategias pedagógicas implementadas desde la clase de educación física, el deporte extraescolar y asignaturas deportivas universitarias sean los espacios donde se impacten en la regulación de conductas agresivas de niños, adolescentes y jóvenes (Orozco Ramírez et al.- (2012), pues se sabe que México es un país que presenta niveles altos de violencia escolar en educación secundaria. Se coincide con Saucedo Ramos y Guzmán Gómez (2018) acerca de que las investigaciones sobre violencia escolar tienen un carácter microsocial y se orientan al análisis de casos específicos o a prácticas en planteles escolares. La violencia entre adultos, es decir, entre profesores, directivos y padres de familia también aqueja a las escuelas y sería importante investigarla para conocer cabalmente lo que sucede en los planteles escolares. Por ello es importante involucrar en estos estudios a los actores principales del proceso de enseñanza-aprendizaje en todos los niveles educativos, es decir; nivel básico, media superior y superior. 


\section{Referencias bibliográficas}

Albaladejo-Blázquez, N. (2011). Evaluación de la violencia escolar en educación infantil y primaria. Ph.D. Tesis Doctoral, Universidad de Alicante, España.

Álvarez-García, D., Núñez Pérez, J. C., \& Dobarro González, A. (2013). Cuestionarios para evaluar la violencia escolar en Educación Primaria y en Educación Secundaria: CUVE3-EP y CUVE3-ESO. Apuntes de Psicología, 31(2), 191-202.

Alvarez García, D., Dobarro, A., Álvarez, L., Núñez, J. C., \& Rodríguez, C. (2014). La violencia escolar en los centros de educación secundaria de Asturias desde la perspectiva del alumnado. Educación XX1, 17(2), 337-360.

Buss, A. H., \& Perry, M. P. (1992). The aggression questionnaire. Journal of Personality and Social Psychology, 63, 452-459.

Conde Vélez, S., \& Delgado-García, M. (2020). Percepciones del alumnado sobre diferentes tipos de violencia. Adaptación y validación del CUVE3-ESO al contexto universitario. Revista de Investigación Educativa, 38(2), 567-581. doi: https://doi.org/10.6018/rie.364431

Cornell, D. G. (2020). Threat assessment as a school violence prevention strategy. Criminology \& Public Policy, 19(1), 235-252. doi: https://doi.org/10.1111/1745-9133.12471

Chen, J. K., \& Chen, L. M. (2020). A Cross-National Examination of School Violence and Nonattendance Due to School Violence in Taiwan, Hong Kong, and Mainland China: A Rasch Model Approach. Journal of School Violence, 19(2), 177-191. doi: 10.1080/15388220.2019.1568882

Chen, Q., Zhu, Y., \& Chui, W. H. (2020). A Meta-Analysis on Effects of Parenting Programs on Bullying Prevention. Trauma, Violence, \& Abuse, 1524838020915619. doi: 10.1177/1524838020915619

Esquivel-Santoveña, E. E., Hernández, R. R., Viveros, N. C., Orozco, F. L., \& van Barneveld, H. O. (2017). Physical Intimate Partner Violence and Controlling Behavior in Mexican University Students and Their Attitudes Toward Social Limits. Journal of Interpersonal Violence, 35(1-2), 403-425. doi: 10.1177/0886260516681879

Ferrara, P., Franceschini, G., Villani, A., \& Corsello, G. (2019). Physical, psychological and social impact of school violence on children. Ital J Pediatr, 45(1), 76. doi: 10.1186/s13052-019-0669-z

Gázquez Linares, J. J., del Carmen Pérez, M., Lucas, F., \& del Mar Palenzuela, M. (2008). Percepción del alumnado universitario sobre el origen de la violencia escolar. European Journal of Education and Psychology, 1(1), 69-80.

Gobierno de los Estados Unidos Mexicanos. (2012). Subsecretaría de Participación Ciudadana y Prevención del Delito. México: Gobierno de los Estados Unidos Mexicanos Retrieved from https://www.ssc.cdmx.gob.mx/organizacion-policial/subsecretaria-de-participacion-ciudadana-yprevencion-del-delito.

Gómez Figueroa, J. A., \& Salazar, C. M. (2015). Detección de violencia escolar en adolescentes en la clase de educación física. Revista Iberoamericana de Psicología del Ejercicio y el Deporte, 10(1), 41-47.

Guerra Vio, C., Álvarez García, D., Dobarro González, A., Núñez Pérez, J. C., Castro Arancibia, L., \& Vargas Castro, J. (2011). Violencia escolar en estudiantes de educación secundaria de Valparaíso (Chile): comparación con una muestra española. Revista Iberoamericana de Psicología y Salud, 2(1), 75-98. 
Hernández, R., Fernández, C., \& Baptista, M. (2010). Metodología de la Investigación (5 ${ }^{\text {th }}$ ed.). México: Mc Graw Hill.

Ingram, K. M., Espelage, D. L., Davis, J. P., \& Merrin, G. J. (2020). Family Violence, Sibling, and Peer Aggression During Adolescence: Associations With Behavioral Health Outcomes. Frontiers in Psychiatry, 11(26). doi: 10.3389/fpsyt.2020.00026

Jenkins, J. H., Sanchez, G., \& Lidia Olivas-Hernández, O. (2020). Loneliness, adolescence, and global mental health: Soledad and structural violence in Mexico. Transcult Psychiatry, 57(5), 673-687. doi: $10.1177 / 1363461519880126$

Kelmendi, K., \& Baumgartner, F. (2017). Exploring Violence Socialization and Approval of Intimate Partner Violence Among University Students in Kosovo. Journal of Interpersonal Violence, 35(5-6), 1081-1107. doi: $10.1177 / 0886260517692336$

Madrigal-Pana, J., Gómez-Figueroa, J., \& Moncada-Jiménez, J. (2018). Adult Perception Toward Videogames and Physical Activity Using Pokémon Go. Games for Health Journal, 8(3), 1-9. doi: 10.1089/g4h.2018.0100

Martínez Vilchis, R., Pozas Rivera, J., Jiménez Arriga, K., Morales Reynoso, T., Aarón Miranda, D., Delgado Maya, M. E., \& Cuenca Sánchez, V. (2015). Prevención de la violencia escolar cara a cara y virtual en bachillerato. Psychology, Society, \& Education, 7(2), 201-212.

Moore, H., Astor, R. A., \& Benbenishty, R. (2020). Role of school-climate in school-based violence among homeless and nonhomeless students: Individual-and school-level analysis. Child Abuse \& Neglect, 102, 104378.

Morales-Vives, F., Codorniu-Raga, M. J., \& Vigil-Colet, A. (2005). Características psicométricas de las versiones reducidas del cuestionario de agresividad de Buss y Perry. Psicothema, 17(1), 96-100.

Orozco Ramírez, L. A., Ybarra Sagarduy, J. L., \& Guerra López, V. (2012). Adaptación del Cuestionario de Violencia Escolar en estudiantes de educación secundaria de México. Revista mexicana de investigación en psicología, 4(1), 14-22.

Park, S., Chiu, W., \& Won, D. (2017). Effects of physical education, extracurricular sports activities, and leisure satisfaction on adolescent aggressive behavior: A latent growth modeling approach. PLOS ONE, 12(4), e0174674. doi: 10.1371/journal.pone.0174674

Polanin, J., Espelage, D., \& Grotpeter, J. (2020). The consequences of school violence: A systematic review and meta-analysis. USA: National Criminal Justice Reference Service Retrieved from https://www.ncjrs.gov/pdffiles1/nij/grants/254672.pdf.

Prieto García, M. P. (2005). Violencia escolar y vida cotidiana en la escuela secundaria. Revista Mexicana de Investigación Educativa, 10(27), 1005-1026.

Ramos-Rodríguez, I., \& Aranda-Beltrán, C. (2020). Violencia y acoso escolar: diferencias por sexo y edad en estudiantes universitarios mexicanos. CIENCIA UNEMI, 13(34), 84-93.

Rodríguez Abrahantes, T. N., Ramos Fernández, O., Rodríguez Abrahantes, A., Larrosa Nogueira, A., \& Ledón Mora, M. (2015). Violencia escolar en adolescentes de una Escuela Secundaria Básica en el Campo, 20142015. Revista Cubana de Enfermería, 31(1), 39-49.

San Román-Mata, S., Puertas-Molero, P., Ubago-Jiménez, J. L., \& González-Valero, G. (2020). Benefits of Physical Activity and Its Associations with Resilience, Emotional Intelligence, and Psychological Distress in 
University Students from Southern Spain. International Journal of Environmental Research and Public Health, 17(12), 4474. doi: https://doi.org/10.3390/ijerph17124474

Saucedo Ramos, C. L., \& Guzmán Gómez, C. (2018). La investigación sobre la violencia escolar en México: tendencias, tensiones y desafíos. Cultura y Representaciones Sociales, 12(24), 213-245.

Shachar, K., Ronen-Rosenbaum, T., Rosenbaum, M., Orkibi, H., \& Hamama, L. (2016). Reducing child aggression through sports intervention: The role of self-control skills and emotions. Children and Youth Services Review, 71, 241-249. doi: https://doi.org/10.1016/j.childyouth.2016.11.012

Stamatis, A., Grandjean, P., Morgan, G., Padgett, R. N., Cowden, R., \& Koutakis, P. (2020). Developing and training mental toughness in sport: a systematic review and meta-analysis of observational studies and pre-test and post-test experiments. BMJ Open Sport \&amp;amp; Exercise Medicine, 6(1), e000747. doi: 10.1136/bmjsem-2020-000747

Taylor, J. (2020). School Violence Prevention: Examining the Impact of Social Emotional Learning Programs. Publich Health Master's, Georgetown University.

Trajković, N., Madić, D. M., Milanović, Z., Mačak, D., Padulo, J., Krustrup, P., \& Chamari, K. (2020). Eight months of school-based soccer improves physical fitness and reduces aggression in high-school children. Biology of sport, 37(2), 185-193. doi: 10.5114/biolsport.2020.94240

Trajković, N., Pajek, M., Sporiš, G., Petrinović, L., \& Bogataj, Š. (2020). Reducing Aggression and Improving Physical Fitness in Adolescents Through an After-School Volleyball Program. Frontiers in Psychology, 11(2081). doi: 10.3389/fpsyg.2020.02081

Vázquez Valls, R., Villanueva Mercado, A. E., Rico, A. F., \& Ramos Herrera, M. A. (2005). La comunidad de la preparatoria 2 de la Universidad de Guadalajara. Actitudes de sus miembros respecto de la violencia y noviolencia escolar. Revista Mexicana de Investigación Educativa, 10(27), 1047-1070.

Esta obra está bajo una Licencia Creative Commons Attribución-NoCommercial 4.0 International

\section{(cc) $\mathrm{EY}$-NC}

